\title{
LOCAL CONNECTIVITY IN HOMEOMORPHISM GROUPS
}

\author{
BY G. S. MCCARTY, JR.
}

Communicated by R. H. Bing, February 28, 1961

Recently there has been increasing interest in the local connectivity of the group of all homeomorphisms of a manifold with boundary. Available tools of proof, however, seem to favor the case of a compact manifold $[1 ; 2]$ or else the use of the topology of uniform convergence of the group [3]. The present note extends such results to the groups of homeomorphisms of certain noncompact manifolds, furnished with the compact-open topology (also see [4]).

If $X$ is a manifold with boundary, let $G(X)$ be the group of homeomorphisms of $X$, with compact-open topology. $G(X)$ is then a topological transformation group on $X$. Let the statement that a space $X$ is (respectively) locally connected, locally contractible or locally $n$-connected be abbreviated by the phase " $X$ is $P_{h}$ ", $h=1,2$ or 3 respectively.

THEOREM. Let $X$ be a compact, connected, Hausdorff manifold with boundary, $\operatorname{dim}(X)>1$, and let $F$ be a finite set of nonboundary points of $X$. If $G(X)$ is $P_{h}, h=1,2$ or 3 , then $G(X-F)$ is $P_{h}$.

In particular, this combines with the results of Hamstrom and Dyer [1] to show that if $\operatorname{dim}(X)=2$ then $G(X-F)$ is locally contractible; and with the results of Hamstrom [2] to show that if $\operatorname{dim}(X)=3$ then $G(X-F)$ is locally $n$-connected, for all $n$.

The following lemma will be used in the proof:

Lemma. Let $X$ be a compact, connected, Hausdorff manifold with boundary, $\operatorname{dim}(X)>1$; and let $F$ be a finite set of nonboundary points of $X$. Then $G(X-F)$ is topologically isomorphic to $G(X, F)$ $=\{g \in G(X): g \mid F \in G(F)\}$.

The proof of the lemma is an exercise in the compact-open topology; the hypothesis that $X$ is a manifold is used in an application of the Jordan-Brouwer theorem. This proof is too long to be given here; it will appear elsewhere in another connection.

Proof OF THE THEOREM. Induction will be used on the number of points of $F$. Let $Y$ be the set of nonboundary points of $X$, and let $\left\{x_{j}\right\}$ be a sequence of distinct points of $Y$. Define $F_{i}=U_{j=1}^{i}\left\{x_{j}\right\}$ and $G_{i}=\left\{g \in G(X): g\left(x_{j}\right)=x_{j}\right.$ if $\left.x_{j} \in F_{i}\right\}$, with the relative topology.

(i) $G_{i}$ is a principal fiber bundle over $Y-F_{i}$ with projection $p: G_{i} \rightarrow Y-F_{i}: g \rightarrow g\left(x_{i+1}\right)$ and fiber $G_{i+1}$, for $i=0,1, \cdots$. The proof of this fact uses the bundle structure theorem: $G_{i+1}$ is a closed subgroup of $G_{i}$, and $G_{i}$ will be a bundle over $G_{i} / G_{i+1}$ if $G_{i+1}$ has a local 
cross-section in $G_{i}$. Furthermore, if the map $p$ is open, then $G_{i} / G_{i+1}$ is homeomorphic to the domain of transitivity of $G_{i}$, namely $Y-F_{i}$. Choose a neighborhood $N$ of $x_{i+1}$ and a homeomorphism of $N$ with a Euclidean space $E^{k}$. The translations of $E^{k}$ provide a set of homeomorphisms of $N$, exactly one of which takes $x_{i+1}$ to each $y \in N$. These maps are all extendible by the identity map outside $N$ to homeomorphisms of $X-F_{i}$; the extensions provide a cross-section in $G_{i}$ above $N$, and $p$ is open.

(ii) If $G_{i}$ is $P_{h}, h=1,2$ or 3 , then $G_{i+1}$ is $P_{h}$; this is an instance of the general remark that, if $F$ is the fiber of an arbitrary bundle $E, F$ is $P_{h}$ if $E$ is $P_{h}$. The following argument, however, uses the instant notation. Choose a neighborhood $N$ of $x_{i+1}$ such that $p^{-1}(N)$ is homeomorphic to the product $N \times G_{i+1}$ : rename the points of $p^{-1}(N)$ using this homeomorphism, and let $q: N \times G_{i+1} \rightarrow G_{i+1}$ be the coordinate projection. Let $U$ be a neighborhood of 1 in $G_{i+1} ; N \times U$ is a neighborhood of 1 in $G_{i}$.

If $G_{i}$ is locally connected, choose a connected neighborhood $V$ of 1 , $V \subset N \times U$; then $q(V) \subset U$ is a connected neighborhood of 1 in $G_{i+1}$. If $G_{i}$ is contractible, choose a neighborhood $V \subset N \times U$ of 1 and a contraction $H: V \times I \rightarrow N \times U$; then $\bar{H}:\left(V \cap G_{i+1}\right) \times I \rightarrow U:(v, t)$ $\rightarrow(q \circ H)(v, t)$ is a contraction of $V \cap G_{i+1}$. A similar construction works in case $G_{i}$ is locally $n$-connected.

(iii) Define $G\left(X, F_{i}\right)=\left\{g \in G(X): g \mid F_{i} \in G\left(F_{i}\right)\right\}$ with the relative topology. Choose open neighborhoods $N_{j}$ for each $x_{j} \in F_{i}$ such that $N_{j} \cap N_{k}=\varnothing$ if $j \neq k$; then $G_{i}=\left\{g \in G\left(X, F_{i}\right): g\left(x_{j}\right) \in N_{j}\right.$ if $\left.x_{j} \in F_{i}\right\}$ which is an open set in $G\left(X, F_{i}\right)$. Hence $G_{i}$ is $P_{h}, h=1,2$ or 3, iff $G\left(X, F_{i}\right)$ is also $P_{h}$.

(iv) By the lemma, $G\left(X, F_{i}\right)$ is topologically isomorphic to $G\left(X-F_{i}\right)$. Thus if $G(X)=G_{0}$ is $P_{h}, h=1,2$ or 3 , then $G_{i}, G\left(X, F_{i}\right)$ and finally $G\left(X-F_{i}\right)$ have been shown to be $P_{h}$.

REMARK. It may be possible to prove by similar methods a like theorem when boundary points are deleted or when $X$ is not connected. The methods are worthless, however, if $F_{i}$ is not discrete.

\section{REFERENCES}

1. M.-E. Hamstrom and E. Dyer, Regular mappings and the space of homeomorphisms on a 2-manifold, Duke Math. J. vol. 25 (1958) pp. 521-531.

2. M.-E. Hamstrom, Regular mappings and the space of homeomorphisms on a 3manifold, Abstract 564-39, Notices Amer. Math. Soc. vol. 6 (1959) p. 783.

3. J. M. Kister, Isotopies in 3-manifolds, Trans. Amer. Math. Soc. vol. 97 (1960) pp. 213-224.

4. D. E. Sanderson, Isotopy in 3-manifolds. III. Connectivity of spaces of homeomorphisms, Proc. Amer. Math. Soc. vol. 11 (1960) pp. 171-176.

University of California at los ANgeles 